\title{
Evasão de estudantes universitários: diagnóstico a partir de dados acadêmicos e socieconômicos
}

\author{
Túlio Albuquerque Pascoal ${ }^{1}$, Daniel Miranda de Brito ${ }^{1}$, \\ Leandro Paiva Andrade $^{1}$, Thaís Gaudencio do Rêgo ${ }^{1}$ \\ ${ }^{1}$ Universidade Federal da Paraíba (UFPB) \\ Caixa Postal 5.115 - 58.051-970 - João Pessoa - PB - Brasil \\ \{tuliopascoal,britmb, leandropiox,gaudenciothais\}@gmail.com
}

\begin{abstract}
Educators and managers of higher education institutions have been concerned about the high dropout rates of their students. Thus, means of early diagnosis of students prone to evasion are desired, in order to avoid that to happen. In this paper, it is proposed an approach to assist institutions in making decisions to combat this phenomenon, using academic and socioeconomic data collected from the students. The viability of the strategy was studied based on records of Computer Science students at UFPB. The results obtained were significant, with accuracy rates of more than $85 \%$ in the classification of students.
\end{abstract}

\begin{abstract}
Resumo. Educadores e gestores das instituições de ensino superior têm se preocupado com os altos índices de evasão de seus estudantes. Dessa forma, meios para o diagnóstico precoce de estudantes propensos à evasão são desejados, de forma a evitá-la. Neste trabalho, propõe-se uma abordagem para auxiliar as instituições nas tomadas de decisão para combate desse fenômeno, a partir do uso de dados acadêmicos e socioeconômicos dos estudantes. A viabilidade da estratégia foi estudada com base em registros de alunos do curso de Ciência da Computação da UFPB. Os resultados obtidos foram relevantes, com taxas de acerto de mais de $85 \%$ na classificação dos estudantes.
\end{abstract}

\section{Introdução}

A evasão no ensino superior é um problema internacional e impacta diretamente a eficiência dos sistemas educacionais [Silva Filho et al. 2007]. Caracterizada como a interrupção dos ciclos de estudo, gera prejuízos significativos em vários aspectos, tais como: sociais, acadêmicos e econômicos [Aparecida et al. 2011] [Baggi e Lopes 2010]. No setor público, as perdas provocadas por estudantes que evadem de seus cursos representam recursos públicos investidos na educação sem o retorno apropriado para a sociedade, enquanto no setor privado acarreta importantes perdas de receita. Em ambos os casos a evasão é fonte de inocupação de pessoal e de equipamentos [Silva Filho et al. 2007].

[Barroso e Falcão 2004], em seu estudo com alunos do curso de Física da Universidade Federal do Rio de Janeiro (UFRJ) discutem a evasão segundo três enfoques distintos: econômico, vocacional e institucional. O primeiro diz respeito à impossibilidade de manutenção do vínculo do aluno por questões socioeconômicas; o segundo se refere à evasão em termos de uma escolha inadequada por parte do aluno, tornando-o desmotivado com a área de graduação; e, por fim, a evasão institucional tem relação com o fracasso nas disciplinas iniciais do curso, seja por inadequação dos métodos de estudo, 
V Congresso Brasileiro de Informática na Educação (CBIE 2016)

Anais do XXVII Simpósio Brasileiro de Informática na Educação (SBIE 2016)

ou por deficiências prévias de conteúdo, impedindo que a aprendizagem ocorra de forma efetiva. Na direção da ideia do primeiro enfoque, a desistência dos alunos também pode ocorrer em virtude de assuntos particulares à vida do estudante, como nos casos em que ingressam no mercado de trabalho e, com isso, experimentam dificuldades em conciliar os horários de estudos com os do emprego [Gisi 2006] [de Souza 2008].

Dentre as áreas que apresentam maiores taxas de evasão, Ciências, Matemática e Computação evidenciam-se, com taxas bem acima da média nacional [Silva Filho et al. 2007]. Em relação aos cursos de Computação, é comum, nas universidades brasileiras, que seus estudantes apresentem dificuldades no aprendizado de disciplinas de programação e lógica, levando a um alto índice de reprovação nas disciplinas específicas da área e, em alguns casos, a desistência do curso [Prietch e Pazeto 2010]. [Silva Filho et al. 2007] constataram que a evasão ocorre com frequência de duas a três vezes maior nos primeiros períodos do curso, portanto, estratégias que visem solucionar o problema da evasão em estágios iniciais do curso tendem a ser adequadas.

Tendo em vista a importância do controle do fenômeno da evasão para a manutenção dos sistemas de ensino de nível superior, apresenta-se neste trabalho, com auxílio de técnicas de mineração de dados educacionais (EDM, do inglês educational data mining), uma abordagem para a identificação de estudantes propensos a evasão, com base nos seus dados socioeconômicos e de desempenho, de modo que educadores possam tomar decisões apoiados no sistema.

A organização do restante do texto é a seguinte: na Seção 2 apresentam-se e discutem-se os trabalhos relacionados encontrados na literatura; na Seção 3 explica-se e detalha-se a metodologia da abordagem proposta e as métricas de avaliação são definidas; a discussão e análise dos resultados obtidos encontram-se na Seção 4 e finalmente, na Seção 5 tem-se a conclusão e possíveis trabalhos futuros.

\section{Trabalhos Relacionados}

Define-se EDM como uma disciplina emergente, preocupada com a criação de estratégias para investigar os diversos tipos de informações originárias de ambientes educacionais, de forma que se possa ter uma melhor compreensão dos estudantes e do sistema educacional em que eles aprendem [Baker et al. 2010]. Assim, a sua utilização permite um melhor entendimento dos dados originados pelo ambiente educacional, através da geração de conhecimentos adicionais, como descoberta de padrões e correlações implícitas nos dados. Portanto, a EDM pode ser utilizada para auxiliar as tomadas de decisão e planejamento dos sistemas de educação, beneficiando todos os afetados direta ou indiretamente (instituição, coordenadores, professores, estudantes, etc.) [Romero e Ventura 2007]. Na Figura 1, é apresentada uma visão geral de como a EDM se relaciona e pode beneficiar os sistemas educacionais.

O interesse nas pesquisas sobre a utilização de métodos de mineração de dados no campo educacional é crescente, principalmente naquelas que tratam do tema da evasão no ensino superior. Nos últimos anos, essas pesquisas tornaram-se evidentes, sejam as desenvolvidas no âmbito das instituições de ensino nacionais, sejam no das instituições internacionais. Ademais, os estudos são desenvolvidas tanto em ambientes presenciais, quanto em ambientes a distância [Romero e Ventura 2010]. [Vitelli et al. 2011] realizaram um estudo sobre evasão de estudantes na Universidade do Vale do Rio dos Sinos 


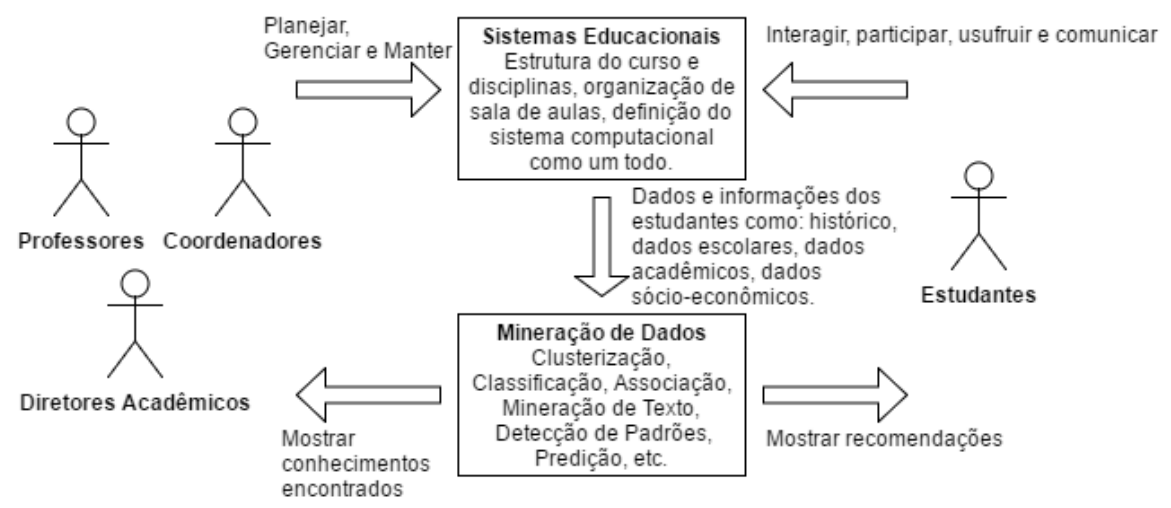

Figura 1. Ciclo de aplicação da Mineração de Dados Educacionais. Adaptado de: [Romero e Ventura 2007] pg. 136

utilizando dados socioeconômicos, desempenho no vestibular, média de desempenho nas atividades, entre outros. Foram aplicados métodos de regressão logística para indicar os fatores que ocasionavam a evasão e quantificar sua relevância. Os resultados revelaram que os fatores mais impactantes estão relacionados ao desempenho acadêmico e ao tempo de curso.

[de Brito et al. 2014] examinam a probabilidade de predição de desempenho de alunos do primeiro semestre do curso de Ciência da Computação da UFPB, utilizando as notas de ingresso na instituição como base. Com o uso de classificadores, os autores alcançaram taxas de acerto de até $75 \%$. Eles argumentam que a identificação do desempenho de alunos nas disciplinas do primeiro período é útil para combater a evasão produzida pelo fracasso nas disciplinas iniciais. Ainda utilizando o ambiente da UFPB, [Pascoal et al. 2015] propuseram um método para previsão de desempenho de alunos de Computação em disciplinas de programação. Foram utilizados quatro algoritmos de aprendizagem de máquina distintos, os quais produziram taxas de acerto sempre acima de $70 \%$, chegando a 84,7\% no melhor caso, com o algoritmo Random Forest.

No estudo de caso de [Dekker et al. 2009], descrevem-se os resultados obtidos na previsão de evasão de estudantes do curso de Engenharia Elétrica, da Universidade de Eindhover. Os autores consideram como critério de predição a educação pré-universitária e as notas dos estudantes no primeiro semestre do curso. Utilizando a ferramenta Weka, os autores conseguiram resultados com taxas de acerto entre $75 \%$ e $80 \%$ na identificação de estudantes com risco de evasão. Em outra pesquisa, [Osmanbegović e Suljić 2012] aplicaram três algoritmos supervisionados de mineração de dados: Naive Bayes, rede neural e árvore de decisão, para prever o sucesso ou o fracasso dos alunos em um curso e avaliaram o desempenho preditivo dos métodos de aprendizagem com base em sua precisão da previsão, facilidade de aprendizagem, e as características de fácil utilização. Os resultados indicaram que o classificador Naive Bayes supera, por sua precisão da previsão, os outros métodos considerados.

[Silva et al. 2014] realizaram um estudo em EDM utilizando regras de associação, a fim de encontrar padrões de regras entre os resultados das provas e os questionários socioeconômicos do Exame Nacional do Ensino Médio (ENEM). Os autores concluíram que 
V Congresso Brasileiro de Informática na Educação (CBIE 2016)

Anais do XXVII Simpósio Brasileiro de Informática na Educação (SBIE 2016)

uma baixa renda familiar, escolaridade dos pais restrita ao nível primário e quantidade elevada de pessoas que residem com os alunos são fatores que reduzem o seu desempenho na prova. Considerando a problemática da evasão, [de Oliveira Júnior et al. 2014] analisaram a correlação entre o empréstimo de livros na biblioteca e a permanência do aluno na instituição. Também foram utilizadas informações de desempenho no ENEM (forma de ingresso na instituição) e informações sociais de gênero e idade. Os autores observaram uma correlação próxima a $80 \%$ entre os atributos de entrada e a situação final do aluno no curso.

\section{Metodologia}

\subsection{Método proposto}

Com intuito de otimizar os sistemas de ensino, objetiva-se neste trabalho disponibilizar uma metodologia para a identificação precoce de estudantes propensos à evasão, de modo a facilitar ações dos educadores que visem reduzir os índices de evasão e permitir que os alunos permaneçam nos cursos. Estudantes são classificados como propensos à evasão ou possíveis concluintes, segundo informações socioeconômicas e de desempenho acadêmico, e a viabilidade das predições é estudada através de testes na ferramenta Weka. Os detalhes da abordagem são apresentados em seguida.

No método proposto, utiliza-se o algoritmo de classificação Naive Bayes. Optouse por sua escolha, em virtude da sua ampla utilização em diversos trabalhos da área [Bhardwaj e Pal 2012] [Baradwaj e Pal 2012] [Xenos 2004], os quais apresentam bons resultados de classificação. O algoritmo é baseado no teorema de Bayes, que classifica uma instância não rotulada com base na maior probabilidade dela estar associada aos seus atributos de entrada, isto é, da hipótese mais provável. A classificação é fundamentada na distribuição de probabilidade de uma classe, dada a probabilidade condicional de seus atributos, assumindo independência entre eles (Naive) [Ang et al. 2016] [Faceli et al. 2011]. O método pode ser resumido a partir da Equação 1:

$$
h=\operatorname{argmax}_{i} P\left(y_{i}\right) \prod_{j=1}^{d} P\left(\mathbf{x}_{j} \mid y_{i}\right)
$$

O classificador Naive Bayes é considerado descritivo e preditivo, sendo assim, apto e recomendado para realizar classificações preditivas. Além de não necessitar de um grande conjunto de treinamento, sua aplicação é fácil, pois só necessita de uma única passagem pelo conjunto de treinamento e requer somente cálculos simples, como de média, variância e probabilidade [Bhardwaj e Pal 2012]. Além disso, o classificador é robusto, mesmo com a presença de ruído e atributos irrelevantes, e apresenta um bom desempenho em uma grande variedade de domínios [Faceli et al. 2011]. A eficiência e simplicidade do método igualmente são fatores de destaque [Osmanbegović e Suljić 2012].

Na Figura 2, uma visão geral da abordagem proposta é apresentada, em termos do seu fluxo de informações e processos. Primeiramente, um conjunto de dados é analisado e classificado pelo algoritmo Naive Bayes, tendo como resultado um modelo (hipótese) criado a partir da natureza dos dados e gerado pelo algoritmo de classificação. Uma vez em posse desse método e dos dados acadêmicos e socioeconômicos de um estudante, os interessados podem utilizar a hipótese aprendida para produzir um rótulo de classe para 
V Congresso Brasileiro de Informática na Educação (CBIE 2016)

Anais do XXVII Simpósio Brasileiro de Informática na Educação (SBIE 2016)

aquele aluno, ou seja, se ele tem risco de evasão no curso ou não. Dada a qualidade dessa previsão, tomadas de decisões e ações para evitar a evasão serão facilitadas (caso sejam necessárias) para os envolvidos no sistema educacional. Uma vez que, por exemplo, o quanto antes um coordenador de curso souber da chance de um de seus alunos evadir, medidas mais efetivas podem ser tomadas para evitar o seu abandono. A última etapa do método, mas não menos importante, é a incorporação dos dados do aluno (juntamente com a classificação resultante do modelo) ao conjunto de dados do sistema, a fim de manter, após uma nova classificação, um conjunto de dados sempre atualizado, de forma a produzir resultados cada vez mais confiáveis, consistentes e condizentes com o cenário.

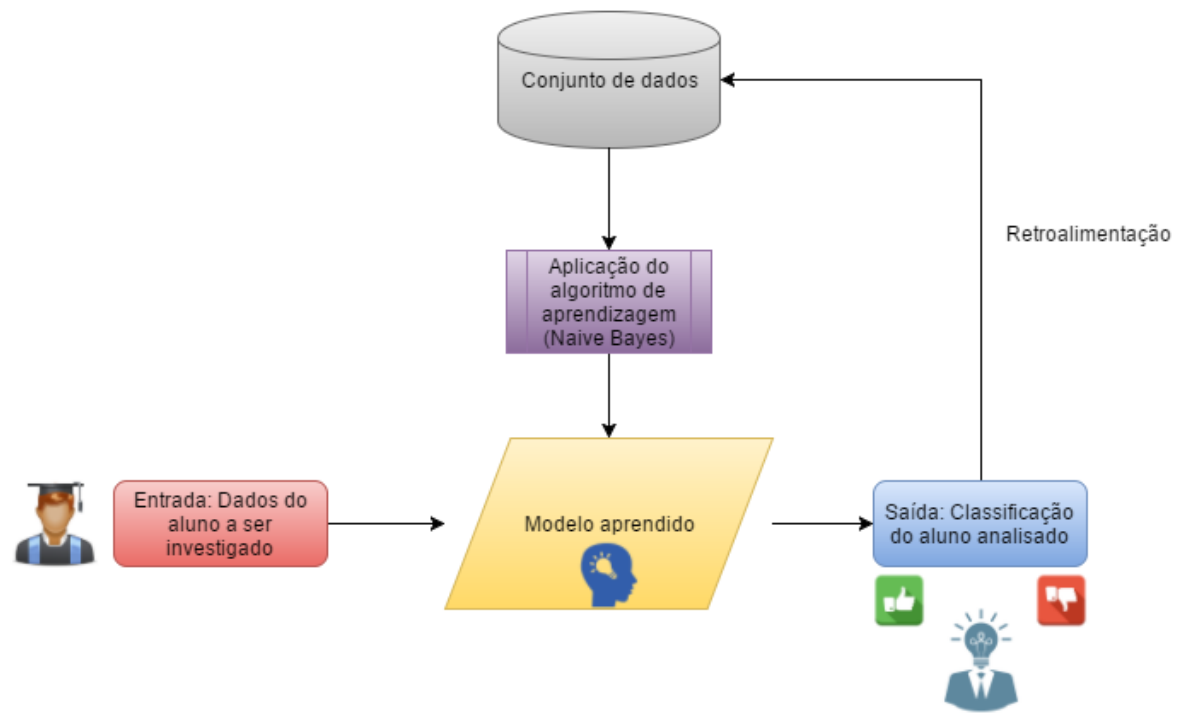

Figura 2. Fluxograma do método proposto

\subsection{Descrição dos dados}

Para estudar a viabilidade da abordagem proposta, utilizaram-se informações referentes a estudantes do curso de Ciência da Computação da Universidade Federal da Paraíba (UFPB). As informações foram cedidas pela Superintendência de Tecnologia da Informação (STI) da Universidade e consistem de registros de estudantes do ano de 2001 a 2013, fornecidos em dois arquivos distintos. O primeiro, contendo os dados referentes às disciplinas cursadas na Universidade e as notas de ingresso (aqui referidas como dados acadêmicos), e a segunda com os dados socioeconômicos dos estudantes. As tabelas foram unidas e o resultado pré-processado, com intuito de eliminar as instâncias com dados ausentes e inconsistentes. Restaram 241 instâncias para análise, sendo 40 da classe concluinte (alunos que concluíram o curso) e 201 da classe evadido (alunos que se evadiram do curso). Para a tarefa de classificação, consideraram-se todos os 22 atributos com valores não ausentes resultantes da união das tabelas, separados em duas modalidades: acadêmicos e socioeconômicos, que podem ser vistos nas Tabelas 1 e 2 , respectivamente.

\subsection{Métricas de avaliação}

A fim de avaliar a qualidade das predições de estudantes, utilizou-se a ferramenta Weka (versão 3.6) [Hall et al. 2009], que provê um método simples para avaliação de algoritmos aprendizagem de máquina [Witten e Frank 2011]. Utilizou-se para a classificação o 
V Congresso Brasileiro de Informática na Educação (CBIE 2016)

Anais do XXVII Simpósio Brasileiro de Informática na Educação (SBIE 2016)

Tabela 1. Atributos acadêmicos levados em consideração pela classificação

\begin{tabular}{|c|c|c|}
\hline Atributo & Descrição & Tipo \\
\hline nota_calculo_1 & Nota obtida na disciplina & Numérico (0 a 10) \\
\hline nota_calculo_vet & Nota obtida na disciplina & Numérico (0 a 10) \\
\hline nota_fisica_1 & Nota obtida na disciplina & Numérico (0 a 10) \\
\hline nota_intro_prog & Nota obtida na disciplina & Numérico 0 a 10 ) \\
\hline nota_intro_comp & Nota obtida na disciplina & Numérico (0 a 10) \\
\hline escola_ens_fund & $\begin{array}{l}\text { Categoria de ensino frequentado } \\
\text { pelo aluno no ensino } \\
\text { fundamental }\end{array}$ & $\begin{array}{l}\text { Nominal (parte em escola particular e parte em escola pública, tendo ficado mais } \\
\text { tempo em escola particular; parte em escola pública e parte em escola particular, } \\
\text { tendo ficado mais tempo em escola pública; somente em escola particular; } \\
\text { somente em escola pública) }\end{array}$ \\
\hline escola_ens_medio & $\begin{array}{l}\text { Categoria de ensino frequentado } \\
\text { pelo aluno no ensino médio }\end{array}$ & $\begin{array}{l}\text { Nominal (parte em escola particular e parte em escola pública, tendo ficado mais } \\
\text { tempo em escola particular; parte em escola pública e parte em escola particular, } \\
\text { tendo ficado mais tempo em escola pública; somente em escola particular; } \\
\text { somente em escola pública) }\end{array}$ \\
\hline turno_ens_medio & $\begin{array}{c}\text { Turno frequentado no ensino } \\
\text { médio }\end{array}$ & $\begin{array}{l}\text { Nominal (integral (dois turnos); parte diurno e parte noturno, predominando o } \\
\text { diurno; parte diurno e parte noturno, predominando o noturno; somente diurno; } \\
\text { somente noturno) }\end{array}$ \\
\hline mediageral & $\begin{array}{l}\text { Média obtida pelo aluno no } \\
\text { exame de admissão na } \\
\text { Universidade }\end{array}$ & Numérico (0 a 1000) \\
\hline
\end{tabular}

algoritmo Naive Bayes. A divisão entre o conjunto de treinamento e testes foi feita pela Validação Cruzada (do inglês Cross- Validation ${ }^{1}$ ) de 10 folds (padrão do Weka).

Como métricas de avaliação de desempenho da abordagem proposta, usou-se a acurácia (número de instâncias classificadas corretamente); matriz de confusão e as métricas dela derivadas (verdadeiros positivos, VP; verdadeiros negativos, VN; falsos positivos, FP; e falsos negativos, FN); e a área sob a curva ROC (AUC, do inglês area under the curve). A matriz de confusão mostra o desempenho de um algoritmo de classificação a partir da relação de qual(is) registro(s) do conjunto de dados foram classificados corretamente. As métricas de VP e VN indicam, respectivamente, classificações corretas dos estudantes que abandonaram e dos estudantes concluíram o curso. Já as taxas de FN e FP correspondem, respectivamente, a estudantes evadidos classificados como concluintes e estudantes concluintes classificados como evadidos. Previamente à análise das classificações, o ganho de informação de cada atributo foi estudado com a finalidade de identificar os mais importantes para a decisão do algoritmo [Witten e Frank 2011].

\section{Discussão dos Resultados}

Nesta seção, apresentam-se os resultados obtidos a partir da análise do conjunto de dados dos alunos, em dois experimentos no Weka. O primeiro analisou a capacidade preditiva de cada atributo para a classificação final do aluno, enquanto o segundo avaliou a eficiência do modelo gerado para a predição de novas instâncias. Inicialmente foram realizados testes somente utilizando os atributos socioeconômicos dos alunos, sem levar em consideração os dados acadêmicos. Adotou-se essa estratégia devido a necessidade de um feedback mais rápido da situação do aluno, tendo em vista que os dados socioeconômicos estão disponíveis ainda antes do aluno iniciar as atividades letivas na instituição. Nesses testes, o algoritmo Naive Bayes obteve uma acurácia total de $78 \%$, porém com taxas de

\footnotetext{
${ }^{1}$ Nesta abordagem o conjunto de dados original é dividido em $k$ subgrupos disjuntos (denominados folds), em que para $k-1$ subgrupos é feito o treinamento do modelo, que é testado com o subconjunto restante. O processo é repetido usando-se subconjuntos diferentes para treinamento e teste, até completar $k$ repetições. Ao término dos testes, calcula-se a média das taxas de acerto e erro de todos os testes.
} 
V Congresso Brasileiro de Informática na Educação (CBIE 2016)

Anais do XXVII Simpósio Brasileiro de Informática na Educação (SBIE 2016)

Tabela 2. Atributos socioeconômicos levados em consideração pela classificação

\begin{tabular}{|c|c|c|}
\hline Atributo & Descrição & Tipo \\
\hline sexo & Sexo do estudante & Nominal (Masculino ou Feminino) \\
\hline cor_pele & Cor da pele do estudante & Nominal (Branca; Negra; Indígena; Amarela; Parda) \\
\hline estado_civil & Estado civil do estudante & Nominal (Solteiro; Casado; Viúvo; Separado; Outro) \\
\hline renda_familiar & Renda familiar do estudante & $\begin{array}{l}\text { Nominal (de } 1 \text { a } 2 \text { salários mínimos; de } 10 \text { a } 20 \text { salários mínimos; de } 2 \text { a } 3 \text { salários } \\
\text { mínimos; de } 20 \text { ou mais salários mínimos; de } 3 \text { a } 5 \text { salários mínimos; de } 5 \text { a } 10 \text { salários } \\
\text { mínimos; menos de } 1 \text { salário mínimo) }\end{array}$ \\
\hline trabalha & $\begin{array}{l}\text { Situação de trabalho (vínculo } \\
\text { empregatício) do estudante }\end{array}$ & $\begin{array}{l}\text { Nominal (não; sim, às vezes; sim, em tempo integral (mais de } 30 \text { horas semanais); sim, } \\
\text { em tempo parcial (até } 30 \text { horas semanais)) }\end{array}$ \\
\hline possui_pc & $\begin{array}{l}\text { Se o estudante possui } \\
\text { computador próprio ou não }\end{array}$ & Nominal (Sim ou Não) \\
\hline acessa_internet & $\begin{array}{l}\text { Se o estudante tem acesso à } \\
\text { Internet }\end{array}$ & Nominal (Sim ou Não) \\
\hline sit_pai & $\begin{array}{l}\text { Situação empregatícia do pai } \\
\text { do estudante }\end{array}$ & $\begin{array}{l}\text { Nominal (é aposentado; está desempregado; está trabalhando; outra; vive de } \\
\text { rendimentos financeiros (aluguéis, aplicações bancárias, etc.;) }\end{array}$ \\
\hline sit_mae & $\begin{array}{l}\text { Situação empregatícia da mãe } \\
\text { do estudante }\end{array}$ & $\begin{array}{l}\text { Nominal (é aposentado; está desempregado; está trabalhando; outra; vive de } \\
\text { rendimentos financeiros (aluguéis, aplicações bancárias, etc.) }\end{array}$ \\
\hline prof_pai & $\begin{array}{l}\text { Tipo de profissão exercida } \\
\text { pelo pai do estudante }\end{array}$ & $\begin{array}{l}\text { Nominal (Alto cargo político ou administrativo e assemelhados; Diretor ou Gerente, } \\
\text { Proprietário de empresa de porte médio e assemelhados; Ocupação desconhecida; } \\
\text { Ocupações do lar e assemelhadas; Profissional Liberal e demais profissões de nivel } \\
\text { superior; Profissões manuais não-especializadas e assemelhadas; Profissões não- } \\
\text { manuais de rotina, Supervisor de trabalho manual, Profissões manuais especializadas e } \\
\text { assemelhados; Proprietário de grande empresa e assemelhados; Supervisor ou inspetor } \\
\text { de ocupações não-manuais, proprietário de pequena empresa e assemelhados) }\end{array}$ \\
\hline prof_mae & $\begin{array}{l}\text { Tipo de profissão exercida } \\
\text { pela mãe do estudante }\end{array}$ & $\begin{array}{l}\text { Nominal (Alto cargo político ou administrativo e assemelhados; Diretor ou Gerente, } \\
\text { Proprietário de empresa de porte médio e assemelhados; Ocupação desconhecida; } \\
\text { Ocupações do lar e assemelhadas; Profissional Liberal e demais profissões de nivel } \\
\text { superior; Profissões manuais não-especializadas e assemelhadas; Profissões não- } \\
\text { manuais de rotina, Supervisor de trabalho manual, Profissões manuais especializadas e } \\
\text { assemelhados; Proprietário de grande empresa e assemelhados; Supervisor ou inspetor } \\
\text { de ocupações não-manuais, proprietário de pequena empresa e assemelhados) }\end{array}$ \\
\hline instr_pai & $\begin{array}{l}\text { Grau de instrução educacional } \\
\text { do pai do estudante }\end{array}$ & $\begin{array}{l}\text { Nominal (Curso universitário completo; Curso universitário incompleto; Ensino } \\
\text { Fundamental (antigo } 1^{\circ} \text { grau) completo; Ensino Fundamental (antigo } 1^{\circ} \text { grau) } \\
\text { incompleto; Ensino Médio ( } 2^{\circ} \text { grau) completo ou equivalente; Ensino Médio }\left(2^{\circ} \text { grau) }\right. \\
\text { incompleto; não frequentou escola; Pós-Graduação (mestrado e/ou doutorado)) }\end{array}$ \\
\hline instr_mae & $\begin{array}{l}\text { Grau de instrução educacional } \\
\text { da mãe do estudante }\end{array}$ & $\begin{array}{l}\text { Nominal (Curso universitário completo; Curso universitário incompleto; Ensino } \\
\text { Fundamental (antigo } 1^{\circ} \text { grau) completo; Ensino Fundamental (antigo } 1^{\circ} \text { grau) } \\
\left.\text { incompleto; Ensino Médio ( } 2^{\circ} \text { grau) completo ou equivalente; Ensino Médio ( } 2^{\circ} \mathrm{grau}\right) \\
\text { incompleto; não frequentou escola; Pós-Graduação (mestrado e/ou doutorado)) }\end{array}$ \\
\hline
\end{tabular}

VN (alunos concluintes classificados corretamente) e FP (alunos concluintes classificados como não concluintes) de $10 \%$ e $90 \%$, com valor da métrica UAC de 0,541 . Valores estes muito aquém do esperado para a proposta de metodologia apresentada. Dessa forma, optou-se por utilizar as informações de desempenho dos estudantes, juntamente com os dados socioeconômicos, a fim de potencializar a eficiência da abordagem, embora a sua aplicação só possa ser feita após o término do primeiro período letivo, quando o aluno já possui as notas das disciplinas iniciais do curso.

A análise do ganho de informação dos atributos do conjunto mostra quanto cada um deles auxilia, individualmente, a classificação final dos estudantes, conforme Tabela 3. Os atributos que obtiveram uma maior influência foram as notas obtidas pelo aluno nas disciplinas do primeiro período do curso. Outra constatação interessante é que as disciplinas de exatas ("Cálculo Vetorial”, "Cálculo Diferencial e Integral I" e "Física Aplicada a Computação I"), que envolvem cálculos matemáticos, influenciam mais na decisão do classificador do que aquelas específicas da área ("Introdução a Programação" e "Introdução ao Computador"). O primeiro conjunto de disciplinas, historicamente, apresenta elevadas taxas de evasão nos cursos de Computação [de Brito et al. 2014], o que pode influenciar de forma decisiva a evasão dos alunos, principalmente naquilo que se refere ao fracasso nas disciplinas iniciais do curso [Barroso e Falcão 2004]. 
V Congresso Brasileiro de Informática na Educação (CBIE 2016)

Anais do XXVII Simpósio Brasileiro de Informática na Educação (SBIE 2016)

\section{Tabela 3. Ranking dos atributos de acordo com seus valores de ganho de informação}

\begin{tabular}{|c|c|}
\hline Atributo & $\begin{array}{c}\text { Valor do ganho de } \\
\text { informação }\end{array}$ \\
\hline nota_calculo_vet & 0,2853 \\
\hline nota_calculo_1 & 0,2617 \\
\hline nota_fisica_1 & 0,2055 \\
\hline nota_intro_comp & 0,1878 \\
\hline nota_intro_prog & 0,1751 \\
\hline instr_mae & 0,0368 \\
\hline sit_mae & 0,0356 \\
\hline instr_pai & 0,0338 \\
\hline prof_mae & 0,0292 \\
\hline cor_pele & 0,0271 \\
\hline estado_civil & 0,0175 \\
\hline
\end{tabular}

\begin{tabular}{|c|c|}
\hline Atributo & $\begin{array}{c}\text { Valor do ganho de } \\
\text { informação }\end{array}$ \\
\hline prof_pai & 0,0162 \\
\hline turno_ens_medio & 0,0156 \\
\hline trabalha & 0,0124 \\
\hline escola_ens_fund & 0,0101 \\
\hline renda_familia & 0,0099 \\
\hline escola_ens_medio & 0,0096 \\
\hline sit_pai & 0,0061 \\
\hline sexo & 0,0022 \\
\hline possui_pc & 0,0010 \\
\hline acessa_internet & 0,0007 \\
\hline mediageral & 0 \\
\hline
\end{tabular}

Percebe-se que o dado social mais importante é o grau de instrução da mãe do aluno (na sétima posição geral do valor de ganho de informação dos atributos), seguido por situação empregatícia da mãe, grau de instrução do pai e tipo de profissão da mãe. Esses resultados podem levar à conclusão de que dentre os dados socioeconômicos de um estudante, aqueles que se referem ao grau de instrução e profissão de seus pais são os mais importantes, sugerindo existir uma relação de continuidade de interesse acadêmico dentro da família. É possível que os pais que possuem nível de escolaridade superior completo tendam a refletir esse interesse para os seus filhos, que acabam sendo estimulados a concluírem um curso superior. Apesar de terem influência menor, não se pode desconsiderar os atributos de cunho social, pois em muitos países, principalmente no Brasil, existe uma grande desigualdade social, o que acarreta numa diferença de oportunidades e de acesso ao ensino e educação, criando uma disparidade entre alunos dentro de uma mesma instituição acadêmica.

Nesta análise destaca-se o valor (0) obtido pelo ganho de informação do atributo mediageral, que indica que o mesmo não possui importância para a definição da situação final do aluno no curso, impossibilitando a definição de uma fronteira clara entre as classes. Esse fato pode estar relacionado à possibilidade que alunos com bom desempenho na prova de ingresso não tenham identificação com o curso e optem por abandoná-lo, além disso é possível que alunos com desempenho inferior no ingresso, superem as suas limitações e consigam concluir o curso. Uma análise detalhada das notas obtidas na prova de ingresso, considerando isoladamente a média de cada matéria, pode ser interessante para investigar com mais detalhes esse fato. Por não ter importância, o atributo foi eliminado para os testes de classificação.

A segunda análise da estratégia avalia a capacidade de identificação dos estudantes evadidos e concluintes, a partir do algoritmo Naive Bayes. A acurácia total do modelo produzido foi de $85,48 \%$, o que atesta a qualidade geral das predições. As taxas de VP e $\mathrm{VN}$ foram $85,60 \%$ e $85,00 \%$, respectivamente. Os resultados mostram que os estudantes potencialmente evadidos, bem como os estudantes que provavelmente concluirão o curso, são identificados com elevadas taxas de acerto. Os FN e FP foram, respectivamente, de $14,40 \%$ e $15,00 \%$, demostrando uma baixa taxa de erro na identificação dos grupos de estudantes. Por fim, quanto à métrica AUC, obteve-se o valor de 0,919, sendo o mínimo considerado 0,5 e o máximo 1, resultado que corrobora a boa qualidade da abordagem quanto a sua capacidade de diagnóstico de evasão de estudantes do curso de Computação. 
V Congresso Brasileiro de Informática na Educação (CBIE 2016)

Anais do XXVII Simpósio Brasileiro de Informática na Educação (SBIE 2016)

As principais contribuições encontradas na estratégia de predição apresentada neste trabalho são: (1) facilitar a detecção de alunos com risco de evasão no curso de forma mais prévia possível; (2) auxiliar a criação e aplicação de novas ações e políticas educativas a fim de evitar a evasão de alunos propensos a evadir do curso; e (3) prover maior confiança ou estímulo para estudantes de acordo com sua classificação dada pelo algoritmo, levando a um maior comprometimento do mesmo, caso necessário.

\section{Considerações Finais e Trabalhos Futuros}

Este trabalho apresentou uma abordagem para o diagnóstico de evasão de estudantes, a partir de seus dados socioeconômicos e acadêmicos. Como forma de validação do método, um conjunto de 241 registros de estudantes concluintes e evadidos, do curso de Ciência da Computação da UFPB, foi analisado na ferramenta Weka. A relevância de cada atributo para a classificação e a capacidade preditiva do método foram avaliados. Constatou-se que as informações acadêmicas dos estudantes têm maior impacto na identificação na sua situação final do que os dados socioeconômicos. Em relação à predição da evasão de estudantes, o método Naive Bayes apresenta uma acurácia geral de $85,48 \%$, indicando que a metodologia proposta é viável e sensata. Espera-se que o procedimento aqui apresentado possa ser adaptado à realidade de cada instituição de ensino e utilizado por alunos, professores e coordenadores de cursos para um melhor entendimento da evasão e para a criação de políticas de combate ao fenômeno no ensino superior, de maneira que os altos índices do problema sejam combatidos.

Como trabalho futuro, acredita-se que o uso do método pode ser ampliado para cursos de outras áreas do conhecimento (Humanas, Saúde, etc.), de forma a identificar novas relações, padrões, características e conhecimentos inerentes a cada área. No entanto, é preciso realizar uma análise posterior a fim de conferir a manutenção dos resultados obtidos neste trabalho. Outra abordagem seria a aplicação e a inclusão de dados demográficos dos estudantes, com objetivo de encontrar relação entre a distância da sua residência e a universidade e o meio de transporte utilizado pelo mesmo e se essas e outras informações semelhantes influenciariam o método na classificação dos estudantes.

\section{Referências}

Ang, S. L., Ong, H. C., e Low, H. C. (2016). Classification Using the General Bayesian Network. Pertanika Journal of Science \& Technology, 24(1).

Aparecida, C., Baggi, S., e Lopes, D. A. (2011). Evasão e avaliação institucional no ensino superior: uma discussão bibliográfica.

Baggi, C. A. d. S. e Lopes, D. A. (2010). Evasão e avaliação institucional no ensino superior: Uma discussão bibliográfica. 2010.

Baker, R. et al. (2010). Data mining for education. International encyclopedia of education, 7:112-118.

Baradwaj, B. K. e Pal, S. (2012). Mining educational data to analyze students' performance. arXiv preprint arXiv:1201.3417.

Barroso, M. F. e Falcão, E. B. (2004). Evasão Universitária: O caso do Instituto de Física da UFRJ. Encontro Nacional de Pesquisa em Ensino de Física, 9:1-14.

Bhardwaj, B. K. e Pal, S. (2012). Data Mining: A prediction for performance improvement using classification. arXiv preprint arXiv:1201.3418. 
V Congresso Brasileiro de Informática na Educação (CBIE 2016)

Anais do XXVII Simpósio Brasileiro de Informática na Educação (SBIE 2016)

de Brito, D. M., de Almeida Júnior, I. A., Queiroga, E. V., e do Rêgo, T. G. (2014). Predição de desempenho de alunos do primeiro período baseado nas notas de ingresso utilizando métodos de aprendizagem de máquina. In Anais do Simpósio Brasileiro de Informática na Educação, volume 25, page 882.

de Oliveira Júnior, J. G., Noronha, R. V., e Kaestner, C. A. A. (2014). Análise da correlação da evasão de cursos de graduação com o empréstimo de livros em biblioteca. In Anais dos Workshops do Congresso Brasileiro de Informática na Educação, volume 3, page 601 .

de Souza, S. L. (2008). Evasão no Ensino Superior: Um Estudo utilizando a Mineração de Dados como ferramenta de Gestão do Conhecimento em um Banco de Dados referente à Graduação de Engenharia. Dissertação de Mestrado, Universidade Federal do Rio de Janeiro.

Dekker, G. W., Pechenizkiy, M., e Vleeshouwers, J. M. (2009). Predicting Students Drop Out: A Case Study. International Working Group on Educational Data Mining.

Faceli, K., Lorena, C. A., Gama, J., e Carvalho, André, C. P. L. F. (2011). Inteligência Artificial: Uma abordagem de aprendizado de máquina. Grupo Gen-LTC.

Gisi, M. L. (2006). A Educação Superior no Brasil e o caráter de desigualdade do acesso e da permanência. Diálogo Educacional, Curitiba, 6(17):97-112.

Hall, M., Frank, E., Holmes, G., Pfahringer, B., Reutemann, P., e Witten, I. H. (2009). The WEKA data mining software: an update. ACM SIGKDD explorations newsletter, 11(1):10-18.

Osmanbegović, E. e Suljić, M. (2012). Data mining approach for predicting student performance. Economic Review, 10(1).

Pascoal, T. A., de Brito, D. M., e do Rêgo, T. G. (2015). Uma abordagem para a previsão de desempenho de alunos de Computação em disciplinas de programação. In Nuevas Ideas en Informática Educativa TISE 2015, pages 454-458.

Prietch, S. S. e Pazeto, T. A. (2010). Estudo sobre a Evasão em um Curso de Licenciatura em Informática e Considerações para Melhorias. WEIBASE, Maceió/AL.

Romero, C. e Ventura, S. (2007). Educational data mining: A survey from 1995 to 2005. Expert systems with applications, 33(1):135-146.

Romero, C. e Ventura, S. (2010). Educational data mining: a review of the state of the art. Systems, Man, and Cybernetics, Part C: Applications and Reviews, IEEE Transactions on, 40(6):601-618.

Silva, L. A., Morino, A. H., e Sato, T. M. C. (2014). Prática de mineração de dados no exame nacional do ensino médio. In Anais dos Workshops do Congresso Brasileiro de Informática na Educação, volume 3, page 651.

Silva Filho, R. L. L., Motejunas, P. R., Hipólito, O., e Lobo, M. (2007). A evasão no ensino superior brasileiro. Cadernos de Pesquisa, 37(132):641-659.

Vitelli, R. F., Rocha, C. S., e Fritsch, R. (2011). Estudo sobre evasão nos cursos de graduação de uma Instituição de Ensino Superior privada: aplicação de regressão logística.

Witten, I. H. e Frank, E. (2011). Data Mining: Practical machine learning tools and techniques. Morgan Kaufmann.

Xenos, M. (2004). Prediction and assessment of student behaviour in open and distance education in computers using Bayesian networks. Computers \& Education, 43(4):345359. 